Pacific Journal of Mathematics

RENORMALIZED PRODUCTS OF THE GENERALIZED FREE 


\title{
RENORMALIZED PRODUCTS OF THE GENERALIZED FREE FIELD AND ITS DERIVATIVES
}

\author{
ABEL KLEIN
}

\begin{abstract}
Renormalized products of the generalized free field and its derivatives are shown to exist as continuous sesquilinear forms on the $C^{\infty}$-vectors of the adjusted free Hamiltonian. Smeared in space and time, with some restrictions on the time smearing, and mild restrictions on the generalized free field, they are shown to be densely defined operators, admitting a self-adjoint extension when the smearing is even in time. Lorentz covariance of these products is shown.
\end{abstract}

In $[4,5]$, Segal has given an intrinsic characterization for the renormalized powers of field operators, which were first introduced by G. C. Wick as a procedure for standardizing the removal of infinities from products of field operators. Segal characterizes these "Wick products" by their commutation relations with the field operators plus the requirement of zero vacuum expectation. He also shows their existence and uniqueness in the case of the "neutral scalar free field" associated with a locally compact abelian group with a given energy operator. They have the property that the field operators at a fixed time generate a maximal abelian selfadjoint algebra. We extend Segal's results to the case of a more general field, commonly called the "generalized free field", introduced by Greenberg [2]. In the case of the generalized free field, the field at a fixed time is not an operator in general, and the field operators must be smeared in time as well as in space, and thus the field operators do not commute. Nevertheless, we show that the methods of [5] still apply, and we extend them to products also involving derivatives of the field. In particular, these extend the results of [5] for products of the form : $\varphi^{\prime}(x)^{r} \dot{\varphi}(x)^{s}$ : in the case of the neutral scalar free field over $\boldsymbol{R}^{n}$ with the usual energy operator. The renormalized products of the generalized free field and its derivatives are shown to exist as continuous sesquilinear forms on the $C^{\infty}$-vectors of the adjusted free Hamiltonian. Moreover, under very mild restrictions on the generalized free field, these renormalized products are shown to be densely defined operators having the $C^{\infty}$ vectors of the adjusted free Hamiltonian in its domain, when the smearing in time is done by a function whose Fourier transform goes to zero fast enough at $\infty$, and admits a self-adjoint extension, when the smearing function is also real and even in time. This was done in [6] for powers of the neutral scalar free field. We also show 
Lorentz covariance of these renormalized products.

B. Simon has pointed out to the author that the result on the existence of self-adjoint extensions follows from Wightman [7, Theorem 3, p. 592]. However, in the case of the renormalized products of the generalized free field and its derivatives it is straightforward to prove it directly, without appealing to the $P C T$ theorem.

It should be noted that the renormalized products of the neutral scalar free field have been studied by Wightman and Gårding [8]. Our work is more on the spirit of Segal $[4,5,6]$.

1. Preliminaries. We shall use the convention that the inner product in a complex Hilbert space is antilinear in the first coordinate, and linear in the second.

If $\mathscr{H}$ is a given complex Hilbert space, the corresponding free Weyl process $(\mathscr{K}, W, \Gamma, v)$ consists of

(i) a complex Hilbert space $\mathscr{r}$.

(ii) a continuous map $W$ from $\mathscr{H}$ to the unitary operators on $\mathscr{K}$ satisfying the Weyl relations:

$$
W(z) W\left(z^{\prime}\right)=\exp \left\{-(i / 2) \operatorname{Im}\left(z, z^{\prime}\right)\right\} W\left(z+z^{\prime}\right), \text { for all } z, z^{\prime} \in \mathscr{H} \text {. }
$$

(iii) a continuous unitary representation $U \rightarrow \Gamma(U)$ of the group of all unitary operators on $\mathscr{H}$, such that:

(a) $\Gamma(U) W(z) \Gamma(U)^{-1}=W(U z)$ for all $U$ and $z$.

(b) $d \Gamma(A) \geqq 0$ for any self-adjoint operator $A \geqq 0$ in $\mathscr{H}$, where $d \Gamma(A)$ denotes the self-adjoint generator of the continuous unitary one-parameter group $\Gamma\left(e^{i t A}\right), t \in \boldsymbol{R}$.

(iv) a unit vector $v \in \mathscr{K}$ which is cyclic for the $W(z)$ and such that $\Gamma(U) v=v$ for all $U$.

The foregoing properties determine $(\mathscr{\kappa}, W, \Gamma, v)$ uniquely, within unitary equivalence.

This definition is due to Segal.

2. The generalized free field. Let $M$ denote $n+1$ dimensional Minkowskian space-time, with relativistic scalar product

$$
x \cdot y=x_{0} y_{0}-\vec{x} \cdot \vec{y} .
$$

If $f$ is a function on $M, f(p)=(2 \pi)^{-(n+1) / 2} \int e^{-i p \cdot x} f(x) d x$ will denote its Fourier transform, when it makes sense. If $\mathscr{F}$ is a set of functions on $M$, with Fourier transforms, $\mathscr{F}$ will denote the set of their transforms.

Let $\rho$ be a given Lorentz invariant positive measure over $M$, such that the support of $\rho$ is contained in $\{k \in M \mid k \cdot k>0\}$, and 
$\rho \in \tilde{\mathscr{S}}^{\prime}(M)$, the space of tempered distributions over space time. By $\left[3\right.$, p. 97], $\rho \in \tilde{\mathscr{S}}^{\prime}$ if and only if $\int\left(1+\|k\|^{2}\right)^{-r / 2} d \rho(k)<\infty$ for some $r>0$ where $\|k\|^{2}=k_{0}^{2}+\vec{k} \cdot \vec{k}$. Equivalently,

$$
d \rho(k)=\delta\left(-k^{2}+m^{2}\right) d k d \mu\left(m^{2}\right),
$$

where $\mu$ is a positive measure on $(0, \infty)$, such that

$$
\int\left(1+2 \vec{k}^{2}+m^{2}\right)^{-r / 2}\left(m^{2}+\vec{k}^{2}\right)^{-1 / 2} d \vec{k} d \mu\left(m^{2}\right)<\infty \text { for some } r>0 .
$$

Let $\tilde{\mathscr{H}}=\left\{\tilde{f} \in L_{2}(M, d \rho) \mid \tilde{f}(k)=\overline{\widetilde{f}}(-k)\right.$ for all $k$ in $\left.M\right\}$. $\tilde{\mathscr{H}}$ is a real Hilbert space, with inner product $(\tilde{f}, \widetilde{g})=\int \tilde{\widetilde{f}}(k) \widetilde{g}(k) d \rho(k)$. We make $\tilde{\mathscr{C}}$ into a complex Hilbert space by defining multiplication by $i$ to be the operator $j$ given by $(j \widetilde{f})(k)=i \varepsilon(k) \tilde{f}(k), \varepsilon(k)=1$ if $k_{0}>0$, and -1 if $k_{0}<0$. We can now introduce the complex inner product $\langle\widetilde{f}, \widetilde{g}\rangle=(\widetilde{f}, \widetilde{g})+i(j \widetilde{f}, \widetilde{g})$. More explicitly, if $\widetilde{A}(\widetilde{f}, \widetilde{g})=\operatorname{Im}\langle\widetilde{f}, \widetilde{g}\rangle$, then $\widetilde{A}(\tilde{f}, \widetilde{g})=-i \int \varepsilon(k) \overline{\tilde{f}}(k) \widetilde{g}(k) d \rho(k)$, and $\langle\widetilde{f}, \widetilde{g}\rangle=2 \int \tilde{\widetilde{f}}(k) \widetilde{g}(k) \theta(k) d \rho(k)$, where $\theta(k)=1$ if $k_{0}>0$, and 0 otherwise.

Let $R \mathscr{S}(M)=\{f \in \mathscr{S}(M) \mid f$ real-valued $\}$. Then

$$
R \tilde{\mathscr{S}}(M)=\{f \in \tilde{\mathscr{S}}(M) \mid \tilde{f}(k)=\overline{\widetilde{f}}(-k)\} \text {. }
$$

Moreover, as $\rho \in \widetilde{\mathscr{S}}^{\prime}, \widetilde{\mathscr{S}}(M) \subset L_{2}(M, d \rho)$, and is dense in $L_{2}(M, d \rho)$, so $R \widetilde{\mathscr{S}}(M) \subset \widetilde{\mathscr{H}}$ and dense in it (actually, we have to identify functions in $\widetilde{\mathscr{S}}$ that are equal almost everywhere with respect to $\rho$, but we will leave this understood). As the Fourier transform is an isomorphism from $\mathscr{S}$ onto $\widetilde{\mathscr{S}}$, we can transfer to $R \mathscr{S}(M)$ the Hilbert space structure, and let $\mathscr{H}$ be the completion of $R \mathscr{S}(M)$. Then we can extend the Fourier transform to $\mathscr{H}$, and it is a unitary map from $\mathscr{H}$ onto $\tilde{\mathscr{H}}$. Thus if $f, g \in \mathscr{H},\langle f, g\rangle_{\mathscr{C}}=\langle\tilde{f}, \tilde{g}\rangle_{\tilde{\mathscr{C}}}$, and we will write $A(f, g)=\operatorname{Im}\langle f, g\rangle=\widetilde{A}(\widetilde{f}, \widetilde{g})$.

Definition. The generalized free field associated with $\rho$ is the free Weyl process over $\mathscr{H}$.

Let $(\mathscr{K}, W, \Gamma, v)$ be the free Weyl process over $\mathscr{H}$. Then the field operators $\Phi(f)$ are defined by $e^{i t \phi(f)}=W(t f)$, for $f \in \mathscr{H}$. Then $[\Phi(f), \Phi(g)]^{-}=i A(f, g)$. For $f, g \in R \mathscr{S}(M)$, we have

$$
A(f, g)=-i(2 \pi)^{-(n+1)} \int \varepsilon(k) e^{i k \cdot(x-y)} f(x) g(y) d x d y d \rho(k)
$$

Let $D(x)=-i(2 \pi)^{-(n+1)} \int \varepsilon(k) e^{i k x} d \rho(k) . \quad D(x)$ is not a function, but 
$D(x) \in \mathscr{S}^{\prime}$, as $\rho \in \tilde{\mathscr{S}}^{\prime}$.

Then $A(f, g)=\int D(x-y) f(x) g(y) d x d y$, so if we write $\Phi(f)=$ $\int \varphi(x) f(x) d x,[\varphi(x), \varphi(y)]=i D(x-y)$.

ExAMPLE. The neutral scalar free field with mass $m_{0}$ : let $d \mu\left(m^{2}\right)=\delta\left(m^{2}-m_{0}^{2}\right) d m^{2}$, or, equivalently, let $d \rho(k)=\delta\left(-k^{2}+m_{0}^{2}\right) d k$. Then $\mathscr{H}$ can be identified with the space of solutions of the KleinGordon equation with mass $m$, $\square \varphi=m^{2} \varphi$. In this case, $D(x)=\Delta_{m_{0}}(x)$ where $\Delta_{m_{0}}$ is the solution of the Klein-Gordon equation, in the sense of distributions, satisfying the Cauchy data

$$
\Delta_{m_{0}}(\vec{x}, 0)=0 ; \frac{\partial}{\partial t} \Delta_{m_{0}}(\vec{x}, 0)=\delta(\vec{x}) .
$$

We recall that in the general case, $d \rho(k)=\delta\left(-k^{2}+m^{2}\right) d k d \mu\left(m^{2}\right)$, so $D(x)=\int \Delta_{m}(x) d \mu\left(m^{2}\right)$. That is the way generalized free fields were first introduced by 0 . W. Greenberg in [2], by requiring the field commutation relations to be an integral with respect to the mass of the commutation relations of the neutral scalar free fields.

We would like now to introduce a particular representation of the generalized free field, which we are going to use in the computations. Let $d \rho_{+}(k)=\theta(k) d \rho(k)$, and $\tilde{\mathscr{H}}_{+}=L_{2}\left(M, d \rho_{+}\right)$. Then the mapping $\widetilde{f} \rightarrow \sqrt{2} \theta \widetilde{f}$ is unitary from $\widetilde{\mathscr{H}}_{\text {onto }} \widetilde{\mathscr{H}}_{+}$, and so we can represent the free Weyl process over $\tilde{\mathscr{C}}$ as the free Weyl process over $\tilde{\mathscr{H}}_{+}$, and we are going to use the Fock-Cook representation (see [1]), in which $\mathscr{K}^{\prime}=\sum_{n=0}^{\infty} \oplus \mathscr{K}_{n}$, where $\mathscr{K}_{n}$ is the symmetrized $n$-fold tensor product of $\tilde{\mathscr{H}}_{+}$with itself. In particular, as

$$
\tilde{\mathscr{H}}_{+}=L_{2}\left(M, d \rho_{+}\right), \quad \mathscr{K}_{n}=S L_{2}\left(M^{n}, \Pi_{i=1}^{n} d \rho_{+i}\right),
$$

where $d \rho_{+i}=d \rho_{+}$for $i=1, \cdots, n$, and $S L_{2}$ means the symmetric functions in $L_{2}$. If $w \in \mathscr{K}_{n}$, we will write $w \sim F\left(k_{1}, \cdots, k_{n}\right)$ to mean $w$ corresponds to the square-integrable symmetric function

$$
F\left(k_{1}, \cdots, k_{n}\right) \text {. }
$$

In this representation the annihilation operator $C^{*}(g)$, for $g \in \mathscr{H}$, is given by

$$
\left(C^{*}(g) F\right)\left(k_{1}, \cdots, k_{n}\right)=\sqrt{2} \sqrt{n+1} \int \overline{\widetilde{g}}(k) F\left(k, k_{1}, \cdots, k_{n}\right) d \rho_{+}(k),
$$

for $F \in \mathscr{K}_{n+1}$ (the $\sqrt{2}$ factor comes from the isomorphism between $\tilde{\mathscr{H}}$ and $\left.\tilde{\mathscr{H}}_{+}\right)$. 


\section{Derivatives of the field. The map}

$$
f \in R \mathscr{S} \longrightarrow \Phi(f)=\int \varphi(x) f(x) d x
$$

can be considered as a tempered operator valued distribution, as the topology of $R \mathscr{S}$ is finer then the topology of $\mathscr{H}$. Thus we can define the derivatives of the field in the same way as for distributions. More precisely, let $\partial$ be a linear differential operator with constant coefficients on $M$, and let $\partial^{*}$ be its formal adjoint. We define

$$
\partial \Phi(f)=\Phi\left(\partial^{*} f\right),
$$

for $f \in R \mathscr{S}$. Symbolically,

$$
\partial \Phi(f)=\int \partial \varphi(x) f(x) d x=\int \varphi(x) \partial^{*} f(x) d x .
$$

Then $[\partial \Phi(f), \Phi(g)]^{-}=i A\left(\partial^{*} f, g\right), \quad$ so $\quad[\partial \varphi(x), \varphi(y)]=i \partial_{x} D(x-y)=$ $i \partial D(x-y)$. Recall $D(x) \in \mathscr{S}^{\prime}$, so $\partial D(x)$ is well defined and is again in $\mathscr{S}^{\prime}$.

In $R \widetilde{\mathscr{S}}, \partial$ is just multiplication by a polynomial, which we will denote by $\tilde{\partial}$. On the support of $\rho,|\tilde{\partial}| \leqq c\left|k_{0}\right|^{d}$, where $d$ is the degree of $\partial$ and $c$ is a constant depending on $\partial$, as $k^{2}=k_{0}^{2}-k^{2}>0$.

4. Action of the Lorentz group on $\mathscr{H}$. There is an action of the inhomogeneous Lorentz group on $\mathscr{H}$ by unitary or antiunitary operators, depending on whether time is not or is reversed. Explicitly, translation by $u \in M$ is represented by the unitary operator whose action on $R \mathscr{S}$ takes $f(x) \rightarrow f(x-a)$, and the corresponding action on $\tilde{\mathscr{H}}_{+}$takes $\tilde{f}(k) \rightarrow e^{-i k \cdot a} \widetilde{f}(k)$; the action of a pure Lorentz transformation $\Lambda$ that preserves the direction of time is unitarily implemented by $f(x) \rightarrow f\left(\Lambda^{-1} x\right)$ in $R \mathscr{S}$, and by $\tilde{f}(k) \rightarrow \widetilde{f}\left(\Lambda^{-1} k\right)$ in $\tilde{\mathscr{H}}_{+}$; and if $\tau$ is a pure Lorentz transformation reversing time, it is represented by the antiunitary operator that takes $f(x) \rightarrow f\left(\tau^{-1} x\right)$ in $R \mathscr{S}$, and $\tilde{f}(k) \rightarrow \overline{\widetilde{f}}\left(-\tau^{-1} k\right)$ in $\tilde{\mathscr{H}}_{+}$. These actions are unitary or antiunitary, as $\rho$ is Lorentz invariant. We will call it the natural representation.

5. Renormalized products. Let $\partial_{1}, \cdots, \partial_{r}$ be linear differential operators in $M$ with constant coefficients. We want to define renormalized products of the form $\partial_{1} \varphi(x) \partial_{2} \varphi(x) \cdots \partial_{r} \varphi(x)$. As shown by Segal in $[4,5]$, the right thing to ask is for these renormalized products to behave in an algebraic sense like ordinary products, i.e., behave under commutation like ordinary products, and have zero vacuum expectation. Proceeding formally, we have 


$$
\begin{aligned}
& {\left[\partial_{1} \varphi(x) \partial_{2} \varphi(x) \cdots \partial_{r} \varphi(x), \varphi(y)\right] } \\
= & i \sum_{j=1}^{r} \partial_{1} \varphi(x) \cdots \partial_{j} \widehat{\varphi(x)} \cdots \partial_{r} \varphi(x) \partial_{j} D(x-y) .
\end{aligned}
$$

Thus, if $g \in \mathscr{H}$

$$
\begin{aligned}
& e^{i \Phi(g)} \partial_{1} \varphi(x) \cdots \partial_{r} \varphi(x) e^{-i \phi(g)} \\
= & \partial_{1} \varphi(x) \cdots \partial_{r} \varphi(x) \\
& +\sum_{j=1}^{r} \partial_{1} \varphi(x) \cdots \widehat{\partial_{j}} \widehat{\varphi(x)} \cdots \partial_{r} \varphi(x) \partial_{j}(D * g)(x) \\
& +\sum_{i<j=1}^{r} \partial_{1} \varphi(x) \cdots \partial_{i} \widehat{\varphi(x)} \cdots \widehat{\partial_{j} \varphi(x)} \cdots \partial_{r} \varphi(x) \partial_{i}(D * g)(x) \partial_{j}(D * g)(x) \\
& +\cdots+\partial_{1}(D * g)(x) \cdots \partial_{r}(D * g)(x),
\end{aligned}
$$

where by $D * g$ we mean $\int D(x-y) g(y) d y$. In general this is an element of $\mathscr{H}$, but not necessarily a function on $M$. But with the right restrictions on $g$ it will be a bounded $C^{\infty}$-function.

Proposition 1. Let $g \in \mathscr{H}$ be such that $\widetilde{g} \in L_{1}(M, d \rho)$. Then $D * g$ is well-defined as a bounded continuous function on $M$. Moreover, if $k_{0}^{r} \widetilde{g} \in L_{1}(M, d \rho)$ for all $r=0,1,2, \cdots, D * g$ is a $C^{\infty}$-function.

Proof. If $\widetilde{g} \in L_{1}(M, d \rho), D * g$ is the Fourier-Stieltjes transform of the complex measure $-i \varepsilon(k) \widetilde{g}(k) d \rho(k)$. To show it is continuously differentiable just recall $|\vec{k}| \leqq\left|k_{0}\right|$ on the support of $\rho$.

Definition. For any positive operator $A$ in a Hilbert space, $\mathscr{D}_{\infty}(A)$ will denote the common part of the domains of the $A^{r}, r=$ $1,2, \cdots$ and $\left[\mathscr{D}_{\infty}(A)\right]$ denotes this set as a topological vector space in the topology in which a generic neighborhood of 0 consists of all $x$ such that $\left\|A^{r} x\right\|<\varepsilon$ for $r<r_{0}$, for some $\varepsilon$ and $r_{0}$.

We are now ready to state our first theorem.

THEOREM 1. Let $B$ be a given positive self-adjoint operator in $\mathscr{H}$, such that $B$ is given by multiplication by a function $\beta$ in $\tilde{\mathscr{H}}$, where $\beta^{-1} \in L_{p}(M, d \rho)$ for $p \varepsilon\left[p_{0}, \infty\right]$ for some $p_{0} \geqq 1$, and there exists $a$ constant $c>0$ and a positive rational number $q$ such that

$$
\left|k_{0}\right| \leqq c|\beta(k)|^{q}
$$

on the support of $\rho$. Let $H=d \Gamma(B)$. Then, for any given linear differential operators with constant coefficients $\partial_{1}, \cdots, \partial_{r}$ on $M$, and every $x \in M$, there exists a continuous sesquilinear form $\varphi\left(\partial_{1}, \cdots, \partial_{r} ; x\right)$ 
on $\left[\mathscr{D}_{\infty}(H)\right]$ such that:

(i) the $\operatorname{map}(x, u, w) \rightarrow \varphi\left(\partial_{1}, \cdots, \partial_{r} ; x\right)(u, w)$ is continuous from $M \times\left[\mathscr{D}_{\infty}(H)\right] \times\left[\mathscr{D}_{\infty}(H)\right]$ into $\boldsymbol{C}$.

(ii) $\varphi(\varnothing ; x)(u, w)=\langle u, w\rangle$ for every $u, w \in\left[\mathscr{D}_{\infty}(H)\right]$, where $\varnothing$ stands for an empty set of linear differential operators; and

$$
\varphi\left(\partial_{1}, \cdots, \partial_{r} ; x\right)(v, v)=0 \quad \text { if } r \geqq 1 .
$$

(iii) for arbitrary $g \in \mathscr{D}_{\infty}(B)$,

$$
\begin{aligned}
& \varphi\left(\partial_{1}, \cdots, \partial_{r} ; x\right)\left(e^{-i \Phi(g)} u, e^{-i \phi(g)} w\right) \\
= & \sum_{s=0}^{r} \sum_{j_{1}<j_{2}<\cdots<j_{s}=1}^{r} \varphi\left(\partial_{1}, \cdots, \widehat{\partial_{j_{1}}}, \cdots, \widehat{\partial_{j_{s}}}, \cdots, \partial_{r} ; x\right)(u, w) \partial_{j_{1}}(D * g)(x) \\
& \cdot \partial_{j_{2}}(D * g)(x) \cdots \partial_{j_{s}}(D * g)(x) .
\end{aligned}
$$

Furthermore, the $\varphi\left(\partial_{1}, \cdots, \partial_{r} ; x\right)$ are uniquely determined by these conditions.

Proof. We first notice that if $g \in \mathscr{D}_{\infty}(B)$, then $\widetilde{g} \in L_{1}(M, d \rho)$, for $\widetilde{g}=\beta^{p} \widetilde{g} \beta^{-p}$, so if we take $p$ large enough so that $\beta^{-1} \in L_{2 p}(M, d \rho)$, it follows $\widetilde{g}$ is the product of two functions, each of which is in $L_{2}(M$, $d \rho)$, and thus it is in $L_{1}(M, d \rho)$. Similarly $k_{0}^{s} \tilde{g} \in L_{1}(M, d \rho)$ for all $s=$ $1,2, \cdots$, as $\left|k_{0}\right| \leqq c|\beta|^{q}$ on the support of $\rho$. Thus by Proposition $1, D * g$ is a bounded $C^{\infty}$-function, so condition (iii) has a nonambiguous meaning.

The uniqueness part of the theorem follows from Theorem 3.2 of [5], by the argument that given $\partial_{1}, \cdots, \partial_{r}$, if the products

$$
\varphi\left(\partial_{i_{1}}, \cdots, \partial_{i_{s}} ; x\right)
$$

are unique for $s<r$, and there exists another form with the same properties as $\varphi\left(\partial_{1}, \cdots, \partial_{r} ; x\right)$, then the two forms have a difference which is invariant under the unitary transformations $e^{i \oplus(g)}, g \in \mathscr{D}_{\infty}(B)$, and has zero vacuum expectation.

The proof of the existence proceeds by the establishment of existence and properties of the limit of $\left\langle u,: \partial_{1} \varphi\left(g_{x}\right) \cdots \partial_{r} \varphi\left(g_{x}\right): w\right\rangle$, as $g$ converges to the $\delta$ function, where $g_{x}(y)=g(y-x), w, u \in \mathscr{D}_{\infty}(H)$, and : : is the renormalization map with respect to the normal vacuum studied by Segal in [4, pp. 426-34].

We will divide the proof in several lemmas. But before proceeding with it we need to introduce some notation: we will write

$$
\beta_{m}\left(k_{1}, \cdots, k_{m}\right)=\sum_{i=1}^{m} \beta\left(k_{i}\right) \text {. }
$$

Thus, if $w \in \mathscr{K}_{m} \cap \mathscr{D}(H)$ and $w \sim F\left(k_{1}, \cdots, k_{m}\right)$,

$$
H w \sim \beta_{m}\left(k_{1}, \cdots, k_{m}\right) F\left(k_{1}, \cdots, k_{m}\right) .
$$


LEMmA 1. Let $w \in \mathscr{K}_{n}, u \in \mathscr{K}_{m}, w \sim F\left(k_{1}, \cdots, k_{n}\right), u \sim G\left(k_{1}, \cdots, k_{m}\right)$. Then for every $g \in R \mathscr{S}(M)$,

$$
\begin{aligned}
& \left\langle u,: \partial_{1} \Phi(g) \cdots \partial_{r} \Phi(g): w\right\rangle \\
= & (m \cdot(m-1) \cdots(m-s+1))^{1 / 2}(n \cdot(n-1) \cdots(n-(r-s)+1))^{1 / 2} \\
& \cdot \int \widetilde{g}\left(k_{1}\right) \cdots \widetilde{g}\left(k_{s}\right) \overline{\widetilde{g}}\left(k_{s+1}\right) \cdots \overline{\widetilde{g}}\left(k_{r}\right) P\left(k_{1}, \cdots, k_{r}\right) \bar{G}\left(k_{1}, \cdots, k_{s}, p_{1}, \cdots, p_{m-s}\right) \\
& \cdot F\left(k_{s+1}, \cdots, k_{r}, p_{1}, \cdots, p_{m-s}\right) d \rho_{+}\left(k_{1}\right) \cdots d \rho_{+}\left(k_{r}\right) d \rho_{+}\left(p_{1}\right) \cdots d \rho_{+}\left(p_{m-s}\right),
\end{aligned}
$$

where $P\left(k_{1}, \cdots, k_{r}\right)=\sum_{\sigma} \overline{\tilde{\partial}}_{\sigma(1)}\left(k_{1}\right) \cdots \overline{\tilde{\partial}}_{\sigma(s)}\left(k_{s}\right) \tilde{\partial}_{\sigma(s+1)}\left(k_{s+1}\right) \cdots \tilde{\partial}_{\sigma(r)}\left(k_{r}\right)$, where the sum is over all permutations $\sigma$ of $\{1, \cdots r\}$, if there exists an integer $0 \leqq s \leqq r$ such that $2 s=m+r-n$, and equals zero otherwise.

$$
\begin{aligned}
\text { Proof. } \Phi(g)= & 2^{-1 / 2}\left(C(g)+C^{*}(g)\right)^{-}, \text {so } \\
& \partial \Phi(g)=2^{-1 / 2}\left(C\left(\partial^{*} g\right)+C^{*}\left(\partial^{*} g\right)\right)^{-} .
\end{aligned}
$$

Thus

$$
\begin{aligned}
& : \partial_{1} \Phi(g) \cdots \partial_{r} \Phi(g): \\
= & 2^{-r / 2} \sum_{s=0}^{r} \sum_{\sigma} C\left(\partial_{\sigma(1)}^{*} g\right) \cdots C\left(\partial_{\sigma(s)}^{*} g\right) C^{*}\left(\partial_{\sigma(s+1)}^{*} g\right) \cdots C^{*}\left(\partial_{\sigma(r)}^{*} g\right)
\end{aligned}
$$

Recalling that if $w \in \mathscr{K}_{n}, u \in \mathscr{K}_{m}$, then

$$
\left\langle u, C\left(g_{1}\right) \cdots C\left(g_{8}\right) C^{*}\left(g_{s+1}\right) \cdots C^{*}\left(g_{r}\right) w\right\rangle=0
$$

unless $m-s=n-(r-s)$, and how $C(g)$ acts on $\mathscr{K}_{n}$ in this representation, we get the lemma.

LEMma 2. There exists a constant $A$, and a positive integer $a$, independent of $g$, such that for arbitrary $w, u \in \mathscr{D}_{\infty}(H)$,

$$
\begin{aligned}
& \left\langle u,: \partial_{1} \Phi(g) \cdots \partial_{r} \Phi(g): w\right\rangle \\
= & \int \prod_{i=1}^{r} \widetilde{g}\left(k_{i}\right) K_{u, w}\left(k_{1}, \cdots, k_{r}\right) d \rho_{+}\left(k_{1}\right) \cdots d \rho_{+}\left(k_{r}\right)
\end{aligned}
$$

where

$$
\begin{aligned}
& \int\left|K_{u, w}\left(k_{1}, \cdots, k_{r}\right)\right| d \rho_{+}\left(k_{1}\right) \cdots d \rho_{+}\left(k_{r}\right) \\
\leqq & A\left\|(1+H)^{a} u\right\| \cdot\left\|(1+H)^{a} w\right\| \cdot
\end{aligned}
$$

Proof. Consider first the special case of Lemma 1, i.e.,

$$
w \in \mathscr{K}_{n} \cap \mathscr{D}_{\infty}(H), \quad u \in \mathscr{K}_{m} \cap \mathscr{D}_{\infty}(H) .
$$

Then, by Lemma 1, the conclusion is equivalent to 


$$
\begin{aligned}
& K_{u, w}\left(k_{1}, \cdots, k_{r}\right) \\
= & (m \cdot(m-1) \cdots(m-s+1))^{1 / 2}(n \cdot(n-1) \cdots(n-(r-s)+1))^{1 / 2} \\
& \cdot \mathscr{X}\left(k_{s+1}\right) \cdots \mathscr{X}\left(k_{r}\right) \int P\left(k_{1}, \cdots, k_{r}\right) \bar{G}\left(k_{1}, \cdots, k_{s}, p_{1}, \cdots, p_{m-s}\right) \\
& \cdot F\left(k_{s+1}, \cdots, k_{r}, p_{1}, \cdots, p_{m-s}\right) d \rho_{+}\left(p_{1}\right) \cdots d \rho_{+}\left(p_{m-s}\right),
\end{aligned}
$$

where $\mathscr{Z}(k)=\overline{\widetilde{g}}(k) g(k)^{-1}$ if $\tilde{g}(k) \neq 0$, and 1 otherwise, be integrable, with absolute integral dominated as indicated. To show that, notice $m \cdot(m-1) \cdots(m-s+1) \leqq m^{r}$, and

$$
n \cdot(n-1) \cdots(n-(r-s)+1) \leqq n^{r},
$$

and $P\left(k_{1}, \cdots, k_{r}\right) \leqq C_{1} \beta_{s}\left(k_{1}, \cdots, k_{s}\right)^{d} \beta_{r-s}\left(k_{s+1}, \cdots, k_{r}\right)^{d}$ on the support of $\rho$ for a positive constant $C_{1}$ and positive integer $d$, as

$$
|\vec{k}| \leqq\left|k_{0}\right| \leqq c \beta^{q}(k)
$$

and $\beta \geqq \varepsilon>0$ for some $\varepsilon>0$. Thus it suffices to prove the required estimate for $C_{1} m^{r / 2} n^{r / 2} \beta_{s}\left(k^{\prime}\right)^{d} \beta_{r-s}\left(k^{\prime \prime}\right)^{d} \int \bar{G}\left(k^{\prime}, p\right) F\left(k^{\prime \prime}, p\right) d \rho_{+}(k) d \rho_{+}(p)$ where $k^{\prime}=\left(k_{1}, \cdots, k_{s}\right), \quad k^{\prime \prime}=\left(k_{s+1}, \cdots, k_{r}\right), p=\left(p_{1}, \cdots, p_{m-s}\right)$, and $d \rho_{+}(k)=$ $d \rho_{+}\left(k_{1}\right) \cdots d \rho_{+}\left(k_{s}\right), d \rho_{+}(p)=d \rho_{+}\left(p_{1}\right) \cdots d \rho_{+}\left(p_{m-s}\right)$. By Lemma 4.2 of [5], we can estimate this by

$$
\begin{aligned}
C_{1} & \left\|m^{r / 2} \beta_{s}\left(k^{\prime}\right)^{d}\left(1+\beta_{s}\left(k^{\prime}\right)+\beta_{m-s}(p)\right)^{b} G\left(k^{\prime}, p\right)\right\|_{2} \\
& \cdot\left\|n^{r / 2} \beta_{r-s}\left(k^{\prime \prime}\right)\left(1+\beta_{r-s}\left(k^{\prime \prime}\right)+\beta_{m-s}(p)\right)^{b} F\left(k^{\prime \prime}, p\right)\right\|_{2} \\
\cdot & \sup _{p}\left(\int\left(1+\beta_{s}\left(k^{\prime}\right)+\beta_{m-s}(p)\right)^{-2 b}\left(1+\beta_{r-s}\left(k^{\prime \prime}\right)+\beta_{m-s}(p)\right)^{-2 b}\right. \\
& \left.d \rho_{+}\left(k^{\prime}\right) d \rho_{+}\left(k^{\prime \prime}\right)\right)^{1 / 2} \cdot
\end{aligned}
$$

Noticing that

$$
\begin{aligned}
& \left\|m^{r / 2} \beta_{s}\left(k^{\prime}\right)^{d}\left(1+\beta_{s}\left(k^{\prime}\right)+\beta_{m-s}(p)\right)^{b} G\left(k^{\prime}, p\right)\right\|_{2} \\
\leqq & \left\|m^{r / 2}\left(1+\beta_{s}\left(k^{\prime}\right)+\beta_{m-s}(p)\right)^{b+d} G\left(k^{\prime}, p\right)\right\|_{2} \\
= & \left\|N^{r / 2}(1+H)^{b+d} u\right\| \leqq C_{2}\left\|(1+H)^{b+d+r / 2} u\right\|,
\end{aligned}
$$

as $B \geqq\left\|\beta^{-1}\right\|_{\infty}^{-1}>0$, and similarly for the term in $F$, all that remains to show is that the last term in the estimate is bounded by a constant independently of $m$ and $n$. In fact,

$$
\begin{aligned}
& \int\left(1+\beta_{s}\left(k^{\prime}\right)+\beta_{m-s}(p)\right)^{-2 b}\left(1+\beta_{r-8}\left(k^{\prime \prime}\right)+\beta_{m-s}(p)\right)^{-2 b} d \rho_{+}\left(k^{\prime}\right) d \rho_{+}\left(k^{\prime \prime}\right) \\
\leqq & \int\left(1+\beta_{s}\left(k^{\prime}\right)\right)^{-2 b}\left(1+\beta_{r-s}\left(k^{\prime \prime}\right)\right)^{-2 b} d \rho_{+}\left(k^{\prime}\right) d \rho_{+}\left(k^{\prime \prime}\right),
\end{aligned}
$$

which if $0<s<r$, is less than equal to

$$
\int \beta_{s}\left(k^{\prime}\right)^{-2 b} d \rho_{+}\left(k^{\prime}\right) \int \beta_{r-s}\left(k^{\prime \prime}\right) d \rho_{+}\left(k^{\prime \prime}\right)
$$


which in turn can be bounded by $\left(\int\left(\beta(q)^{-2 b}+\beta(q)^{-2 b / r}\right) d \rho_{+}(q)\right)^{2}$, as we will show, and that is bounded independently of $m$ and $n$, if we choose $b$ large enough so that $2 b / r>p_{0}$, as $\beta^{-1} \in L_{p}$ for $p \in\left[p_{0}, \infty\right]$. It suffices thus to show that

$$
\begin{array}{r}
\int \beta_{j}\left(k_{1}, \cdots, k_{j}\right)^{-2 b} d \rho_{+}\left(k_{1}\right) \cdots d \rho_{+}\left(k_{j}\right) \leqq \int\left(\beta(q)^{-2 b}+\beta(q)^{-2 b / r}\right) d \rho_{+}(q), \\
\text { if } 0<j<r .
\end{array}
$$

But this follows from the inequality

$$
a_{1} a_{2} \cdots a_{j} \leqq\left(a_{1}+a_{2}+\cdots+a_{j}\right)^{j}, \quad \text { if } a_{i}>0, i=1, \cdots, j
$$

and the fact $\beta(q)^{-2 b l j} \leqq \beta(q)^{-2 b}+\beta(q)^{-2 b / r}$, for $0<j<r$.

If $s=0$ or $s=r$, trivialized versions of the above argument are applicable.

Now let $w$ and $u$ be arbitrary in $\mathscr{D}_{\infty}(H)$, and write $w=\sum_{n} w_{n}$, $u=\sum_{m} u_{m}$; where $w_{n}, u_{n} \in \mathscr{K}_{n}$. As $e^{i t I I}$ and $e^{i t N}$ commute, each $w_{n}$ and $u_{m}$ is again in $\mathscr{D}_{\infty}(H)$, and the estimate just derived is applicable. Thus each $K_{u_{m}, w_{n}}$ is integrable, and the question reduces to the integrability of $\sum_{m, n} K_{u_{m}, w_{n}}$, and to the estimate of its $L_{1}$ norm. Now : $\partial_{1} \Phi(g) \cdots \partial_{r} \Phi(g): w_{n}$ is orthogonal to $u_{m}$, in case $|m-n|>r$, so the sum in question can be expressed as $\sum_{s=-r}^{r} \sum_{m} K_{u_{m}, w_{m+s}}$. The sum over $s$ being finite, it suffices to establish the integrability and estimate for $\sum_{m} K_{u_{m}, w_{m+s}}$. It is evidently enough to show

$$
\sum_{m}\left\|K_{u_{m}, w_{m}+s}\right\|_{1}
$$

is finite and satisfies the required inequality. Now

$$
\left\|K_{u_{m}, w_{m+s}}\right\|_{1} \leqq A\left\|(1+H)^{a} u_{m}\right\| \cdot\left\|(1+H)^{a} w_{m+s}\right\|,
$$

so

$$
\begin{aligned}
& \sum_{m}\left\|K_{u_{m}, w_{m}+s}\right\|_{1} \leqq A \sum_{m}\left\|(1+H)^{a} u_{m}\right\| \cdot\left\|(1+H)^{a} w_{m+s}\right\| \\
\leqq & a\left(\sum_{m}\left\|(1+H)^{a} u_{m}\right\|^{2}\right)^{1 / 2}\left(\sum_{n}\left\|(1+H)^{a} w_{n}\right\|^{2}\right)^{1 / 2} \\
= & A\left\|(1+H)^{a} u\right\| \cdot\left\|(1+H)^{a} w\right\| \cdot
\end{aligned}
$$

It follows $\left\|K_{u, w}\right\|_{1} \leqq 2 r A\left\|(1+H)^{a} u\right\| \cdot\left\|(1+H)^{a} w\right\|$, as required.

Lemma 3. The limit $\lim _{g \rightarrow o}\left\langle u,: \partial_{1} \Phi\left(g_{x}\right) \cdots \partial_{r} \Phi\left(g_{x}\right): w\right\rangle$ exists, and is a continuous function of $x \in M, u, w \in\left[\mathscr{D}_{\infty}(H)\right]$.

Proof. As $\widetilde{g}_{x}(k)=e^{-i k \cdot x} \widetilde{g}(k)$, we have, by Lemma 2

$$
\left\langle u,: \partial_{1} \Phi\left(g_{x}\right) \cdots \partial_{r} \Phi\left(g_{x}\right): w\right\rangle=\int \prod_{i=1}^{r} \widetilde{g}\left(k_{1}\right) e^{-i\left(\Sigma_{i=1}^{r} k_{i}\right) \cdot x} K_{u, w}(k) d \rho_{+}(k)
$$


where $k=\left(k_{1}, \cdots, k_{r}\right), d \rho_{+}(k)=d \rho_{+}\left(k_{1}\right) \cdots d \rho_{+}\left(k_{r}\right)$, and

$$
\left\|K_{u, w}\right\|_{1} \leqq A\left\|(1+H)^{a} u\right\|\left\|(1+H)^{a} w\right\|,
$$

$K$ and $a$ being constants independent of $g, u, w$. If $g \rightarrow \delta$, then $\widetilde{g} \rightarrow 1$, and by dominated convergence the limit as $g \rightarrow \delta$ exists and equals $\int e^{-i\left(\Sigma_{1}^{r} k_{i}\right) \cdot x} K_{u, w}(k) d \rho_{+}(k)$. Let us call this limit $\varphi\left(\partial_{1}, \cdots, \partial_{r} ; x\right)$ $\times(u, w)$. Then $\left|\varphi\left(\partial_{1}, \cdots, \partial_{r} ; x\right)(u, w)\right| \leqq A\left\|(1+H)^{a} u\right\|\left\|(1+H)^{a} w\right\|$, and this reduces the question of its continuity as a function of $x, u$, $w$ to the question of its continuity as a function of $x$, and this is clear from its representation above as an absolutely convergent integral.

Conclusion of proof of theorem: We have only to show that $\varphi\left(\partial_{1}, \cdots, \partial_{r} ; x\right)$ satisfies conditions (i), (ii), and (iii). Condition (i) was proved in Lemma 3, (ii) follows from $\left\langle v,: \partial_{1} \Phi(g) \cdots \partial_{r} \Phi(g): v\right\rangle=0$ if $r>0$ for all $g$, together with the definition of $\varphi(\varnothing ; x)$. To prove (iii), notice that

$$
\begin{aligned}
& e^{i \phi(h)}: \partial_{1} \Phi(g) \cdots \partial_{r} \Phi(g): e^{-i \Phi(h)} \\
= & \sum_{s=0}^{r} \sum_{j_{1}<j_{2}<\cdots<j_{s}=1}^{r}: \partial_{1} \Phi(g) \cdots \widehat{\partial_{j_{1}} \Phi(g)} \widehat{\cdots} \widehat{\partial_{j_{s}} \Phi(g)} \cdots \partial_{r} \Phi(g): \\
& \times A\left(\partial_{j_{1}}^{*} g, h\right) \cdots A\left(\partial_{j_{s}}^{*} g, h\right) .
\end{aligned}
$$

This follows from $e^{i \nabla(h)} \Phi(g) e^{-i \phi(h)}=\Phi(g)+A(g, h)$, which follows from the Weyl relations, together with an induction using Theorem 1.3 (a) of [4].

If we now apply the expression above to $w$, and take its inner product with $u$, having substituted $g_{x}$ for $g$, and then take the limit as $g \rightarrow \delta$, we obtain (iii), noticing that $\lim _{g \rightarrow \delta} A\left(\partial^{*} g_{x}, h\right)=\partial(D * h)(x)$, and using $e^{i \phi(h)} w \in \mathscr{D}_{\infty}(H)$, if $h \in \mathscr{D}_{\infty}(B)$ (Theorem 3.2 of [5]). End of proof.

REMARK. As an example of the operator $H$ involved in the theorem, we can take $H$ as the free Hamiltonian, i.e., $\beta(k)=k_{0}$, if $k_{0}$ is bounded away from zero in the support of $\rho$, and otherwise take $\beta(k)=\varepsilon(k)+k_{0}$. In any case we will denote this operator by $H_{0}$, and call it the adjusted free Hamiltonian.

COROLLARY. With the same hypothesis as in the theorem, there exists for every $f \in L_{1}(M)$ and linear differential operators with constant coefficients $\partial_{1}, \cdots, \partial_{r}, a$ continuous sesquilinear form $\Phi\left(\partial_{1}, \cdots, \partial_{r} ; f\right)$ on $\left[\mathscr{D}_{\infty}(H)\right]$ such that

(i) $\Phi\left(\partial_{1}, \cdots, \partial_{r} ; f\right)$ is a linear function of $f$, and the map $(f, u, w) \rightarrow \Phi\left(\partial_{1}, \cdots, \partial_{r} ; f\right)(u, w)$ is continuous from 


$$
L_{1}(M) \times\left[\mathscr{D}_{\infty}(H)\right] \times\left[\mathscr{D}_{\infty}(H)\right]
$$

into $\boldsymbol{C}$.

(ii) $\Phi(\varnothing ; f)(u, w)=\langle u, w\rangle$, where $\varnothing$ stands for an empty set of linear differential operators, and $\Phi\left(\partial_{1}, \cdots, \partial_{r} ; f\right)(v, v)=0$ if $r \geqq 1$.

(iii) For arbitrary $g \in \mathscr{D}_{\infty}(B)$,

$$
\begin{aligned}
& \Phi\left(\partial_{1}, \cdots, \partial_{r} ; f\right)\left(e^{-i \Phi(g)} u, e^{-i \Phi(g)} w\right) \\
= & \sum_{s=0}^{r} \sum_{j_{1}<j_{2}<\cdots<j_{s}=1}^{r} \Phi\left(\partial_{1}, \cdots, \partial_{j_{1}}, \cdots, \partial_{j_{s}}, \cdots, \partial_{r} ; f \partial_{j_{1}}(D * g) \cdots \partial_{j_{s}}(D * g)\right)(u, w)
\end{aligned}
$$

for all $u, w \in\left[\mathscr{D}_{\infty}(H)\right]$.

Furthermore, the $\Phi\left(\partial_{1}, \cdots, \partial_{r} ; f\right)$ are uniquely characterized by these conditions.

Proof. Let $\left.\Phi\left(\partial_{1}, \cdots, \partial_{r} ; f\right)(u, w)=\int \varphi^{\prime} \partial_{1}, \cdots, \partial_{r} ; x\right)(u, w) f(x) d x$. Then (i), (ii), (iii) follow from Theorem 1 , and uniqueness is proved as in the theorem. End of proof.

We have by now established the existence of the renormalized products as continuous sesquilinear forms. We can now look at their existence as operators in $\mathscr{K}$.

Definition. Let $R$ be a densely defined sesquilinear form on a Hilbert space $\mathscr{K}$, with dense domain $\mathscr{D}$. The operator $R_{0 p}$ associated with $R$ will be defined as having domain

$$
\begin{aligned}
\mathscr{D}\left(R_{o p}\right)= & \{w \in \mathscr{D}\|R(u, w) \mid \leqq c(w)\| u \| \text { for all } u \in \mathscr{D}, \\
& \text { where } c(w) \text { is a finite constant }\},
\end{aligned}
$$

and for all $w \in \mathscr{D}\left(R_{o p}\right), R_{o p} w$ is given by $\left\langle u, R_{o p} w\right\rangle=R(u, w)$ for all $u \in \mathscr{D}$. We will also write $R$ for $R_{0 p}$, when it cannot cause confusion.

Proposition 2. Let $\mathscr{F}$ be any class of integrable functions on $M$, which is invariant under multiplication by $\partial_{i}(D * g), i=1, \cdots, r$, $g \in \mathscr{D}_{\infty}(B)$. Then if there exists $w \in \mathscr{D}_{\infty}(H)$ in the domain of

$$
\Phi\left(\partial_{i_{1}}, \cdots, \partial_{i_{s}} ; f\right) \text { for all } f \in \mathscr{F},\left\{\partial_{i_{1}}, \cdots, \partial_{i_{s}}\right\} \subset\left\{\partial_{1}, \cdots, \partial_{r}\right\},
$$

these operators are densely defined.

Proof. Let $\mathscr{D}$ be intersection of the domains of the

$$
\Phi\left(\partial_{i_{1}}, \cdots, \partial_{i_{s}} ; f\right) \text {, for } f \in \mathscr{F},\left\{\partial_{1_{1}}, \cdots, \partial_{1_{s}}\right\} \subset\left\{\partial_{1}, \cdots, \partial_{r}\right\} \text {. }
$$


It suffices to show $e^{-i \phi(g)} \mathscr{D} \subset \mathscr{D}$ for all $g \in \mathscr{D}_{\infty}(B)$, for then $\overline{\mathscr{D}}=\mathscr{K}$ or $\{0\}$, as the $e^{-i \emptyset(g)}, g \in \mathscr{D}_{\infty}(B)$, form an irreducible set of operators.

The proof of $e^{-i \phi(g)} \mathscr{D} \subset \mathscr{D}, g \in \mathscr{D}_{\infty}(B)$, goes by induction, using condition (iii) of Corollary. End of proof.

REMARK. Our next theorem will be to the effect that the $\Phi\left(\partial_{1} \cdots \partial_{r}: ; f\right)$ are indeed densely defined operators, with some mild restrictions on $p$, for a certain class of $f$ 's, and have $\mathscr{D}_{\infty}\left(H_{0}\right)$ on its domain, where $H_{0}$ is the adjusted free Hamiltonian. Another way of getting that kind of result, would be to prove that the vacuum $v$ is in the domain of the $\Phi\left(\partial_{1}, \cdots, \partial_{r} ; f\right)$, for a certain class $\mathscr{F}$, that could be taken as $\mathscr{S}(M)$, which is invariant under the $\partial_{j}(D * g)$, by Proposition 1, and then apply Proposition 2. The proof that $v$ is in all those domains, is contained in the proof of Theorem 2, and we would need condition (ii) of Theorem 2, but (iii) is not needed. Of course, the result is somewhat weaker than Theorem 2, as our operators would have a smaller domain.

THeOREM 2. Let $H_{0}$ be the adjusted free Hamiltonian, i.e., $H_{0}=d \Gamma(B)$, where $B$ acts in $\tilde{\mathscr{H}}$ as multiplication by $\beta$, where $\beta(k)=k_{0}$ in case $\left|k_{0}\right| \geqq \delta>0$ on the support of $\rho$, or $\beta(k)=\varepsilon(k)+k_{0}$ otherwise. Suppose also

( i ) $|\tilde{f}(k)| \leqq g(\vec{k}) h\left(k_{0}\right)$, where $g \in L_{2}\left(\boldsymbol{R}^{n}\right) \cap L_{\infty}\left(\boldsymbol{R}^{n}\right), h \in \mathscr{S}(\boldsymbol{R})$.

(ii) $\int(1+m)^{-q} m^{-1} d \mu\left(m^{2}\right)<\infty$, for some $q \geqq 1$.

(iii) $\int_{0}^{z} m^{-1} d \mu\left(m^{2}\right) \leqq c_{1} z^{c_{2}}$, for some $c_{1}>0, c_{2}>0$.

Then $\mathscr{D}_{\infty}\left(H_{0}\right) \subset \mathscr{D}\left(\Phi\left(\partial_{1}, \cdots, \partial_{r} ; f\right)\right)$ for all linear differential operators with constant coefficients $\partial_{1}, \cdots, \partial_{r}$.

Proof. We have to show $\left|\Phi\left(\partial_{1}, \cdots, \partial_{r} ; f\right)(u, w)\right| \leqq c(w)\|u\|$ for all $u, w \in \mathscr{D}_{\infty}(H)$, where $c(w)<\infty$. It suffices, by the argument given in the end of the proof of Lemma 2, to show

$$
\left|\Phi\left(\partial_{1}, \cdots, \partial_{r} ; f\right)(u, w)\right| \leqq A\left\|(1+H)^{a} w\right\| \cdot\|u\|
$$

for $w \in \mathscr{K}_{n} \cap \mathscr{D}_{\infty}(H), u \in \mathscr{K}_{m} \in \mathscr{D}_{\infty}(H)$, for suitable constants $A$, an, independent of $u, w, n, m$.

From the proof of Theorem 1, with the same notation

$$
\begin{aligned}
& \Phi\left(\partial_{1}, \cdots, \partial_{r} ; f\right)(u, w) \\
= & (2 \pi)^{n+1 / 2} \int \tilde{f}\left(\sum_{1}^{s} k_{i}-\sum_{s+1}^{r} k_{i}\right) K_{u, w}^{\prime}\left(k_{1}, \cdots, k_{r}\right) d \rho_{+}\left(k_{1}\right) \cdots d \rho_{+}\left(k_{r}\right)
\end{aligned}
$$

where 


$$
\begin{aligned}
& K_{u, w}^{\prime}\left(k_{1}, \cdots, k_{r}\right) \\
= & (m \cdot(m-1) \cdots(m-s+1))^{1 / 2}\left(n \cdots(n-(r-s)+1)^{1 / 2} .\right. \\
& P\left(k_{1}, \cdots, k_{r}\right) \int \bar{G}\left(k^{\prime}, p\right) F\left(k^{\prime \prime}, p\right) d \rho_{+}(p), 2 s=m+r-n,
\end{aligned}
$$

if such an $0 \leqq s \leqq r$ exists, or equals zero otherwise. We only need to consider the case when such an $s$ exists. The factors in $m$ and $n$ can be bounded by $A_{1} n^{r}$, where $A_{1}$ is a constant, as

$$
|m-n| \leqq r P\left(k_{1}, \cdots, k_{r}\right) \leqq A_{2} \beta_{s}\left(k^{\prime}\right)^{d} \beta_{r-s}\left(k^{\prime \prime}\right)^{d},
$$

for constants $A_{2}, d$, as in the proof of Lemma 2 .

Thus, using Lemma 4.2 of [5], in the same way as in the proof of Lemma 1, we get

$$
\left|\Phi\left(\partial_{1}, \cdots, \partial_{r} ; f\right)(u, w)\right| \leqq A_{3}\left\|N^{r} H^{d}(1+H)^{b} w\right\|\|u\| Q,
$$

where $A_{3}$ is a suitable constant and

$Q=\sup _{p}\left(\int \beta_{s}\left(k^{\prime}\right)^{d}\left(1+\beta_{r-s}\left(k^{\prime \prime}\right)+\beta_{m-s}(p)\right)^{-2 b}\left|\tilde{f}\left(\sum_{1}^{s} k_{i}-\sum_{s+1}^{r} k_{i}\right)\right|^{2} d \rho_{+}(k)\right)^{1 / 2}$.

It suffices to show $Q$ is bounded with a bound independent of $n, m$, and $0 \leqq s \leqq r$.

Recalling $\beta_{m-s}(p)>0$, we have

$$
\begin{aligned}
Q^{2} & \leqq \int \beta_{s}\left(k^{\prime}\right)^{d} \beta_{r-s}\left(k^{\prime \prime}\right)^{-2 b}\left|\tilde{f}\left([k]_{s}-[k]_{r-s}\right)\right|^{2} d \rho_{+}(k) \\
& \leqq \int \beta_{s}\left(k^{\prime}\right)^{d} \beta_{r-s}\left(k^{\prime \prime}\right)^{-2 b}\left|g\left([\vec{k}]_{s}-[\vec{k}]_{r-s}\right)\right|^{2} \cdot\left|h\left(\left[k_{0}\right]_{s}-\left[k_{0}\right]_{r-s}\right)\right|^{2} d \rho_{+}(k)
\end{aligned}
$$

where $[k]_{s}=\sum_{1}^{s} k_{i},[k]_{r-s}=\sum_{s+1}^{r} k_{i}$.

Since $h \in \mathscr{S}(\boldsymbol{R}),|h(\xi)| \leqq c_{j}(r+(\xi))^{-j}$ for some constant $c_{j}$ for $j=1,2, \cdots$.

We will estimate the last integral in two regions:

I: $\left|\left[k_{0}\right]_{s}-\left[k_{0}\right]_{r-s}\right| \geqq 1 / 2\left[k_{0}\right]_{s}$

II: $\left|\left[k_{0}\right]_{s}-\left[k_{0}\right]_{r \rightarrow s}\right|<1 / 2\left[k_{0}\right]_{s}$.

In region $I$, the integral in question is bounded by

$$
\begin{aligned}
& c_{j}^{2} \int \beta_{s}\left(k^{\prime}\right)^{d}\left(r+\left[k_{0}\right]_{s}\right)^{-2 j} \beta_{r-s}\left(k^{\prime \prime}\right)^{-2 b}\left|g\left([\vec{k}]_{s}-[\vec{k}]_{r-s}\right)\right|^{2} d \rho_{+}(k) \\
\leqq & c_{j}^{2} \int\left(r+\left[k_{0}\right]_{s}\right)^{-2 j+d} \beta_{r-s}\left(k^{\prime \prime}\right)^{-2 b}\left|g\left([\vec{k}]_{s}-[\vec{k}]_{r-s}\right)\right|^{2} d \rho_{+}(k)
\end{aligned}
$$

as $\beta(k)=k_{0}$ or $1+k_{0}$ on the support of $\rho_{+}$.

Recalling $\left(a_{1}+a_{2}+\cdots+a_{n}\right)^{n} \geqq a_{1} a_{2} \cdots a_{n}$, and choosing $j$ such that $-2 j+d<0$, we can bound the above expression by 


$$
\begin{aligned}
& \quad c^{\prime} \int \prod_{1}^{s}\left(1+k_{0_{i}}\right)^{(-2 j+d) / r} \prod_{s+1}^{r} \beta\left(k_{i}\right)^{-2 b / r}\left|g\left([\vec{k}]_{s}-[\vec{k}]_{r-s}\right)\right|^{2} d \rho_{+}(k) \\
& \leqq \\
& c^{\prime \prime} \int \prod_{1}^{r}\left(1+k_{0_{i}}\right)^{-a}\left|g\left([\vec{k}]_{s}-[\vec{k}]_{r-s}\right)\right|^{2} d \rho_{+}(k),
\end{aligned}
$$

for suitable constants $c^{\prime}, c^{\prime \prime}, a<(2 j-d) / r, a<2 b / r$, which as $\rho_{+}$is invariant under the proper Lorentz group, is equal to

$$
\begin{aligned}
& c^{\prime \prime} \int \prod_{1}^{r}\left(1+k_{0_{i}}\right)^{-a}\left|g\left(\sum_{1}^{r} \vec{k}_{i}\right)\right|^{2} d \rho_{+}(k) \\
= & c^{\prime \prime} \int \prod_{1}^{r}\left(1+k_{0_{1}}\right)^{-a}\left|g\left(\sum_{1}^{r} k_{i}\right)^{2}\right| \prod_{1}^{r} \frac{\delta\left(k_{0_{i}}-\sqrt{m_{i}^{2}+\vec{k}_{i}^{2}}\right.}{2 k_{0_{i}}} d k_{0_{i}} d \vec{k}_{i} d \mu\left(m_{i}^{2}\right) \\
= & c^{\prime \prime \prime} \int\left(\int \prod_{1}^{r}\left(1+\sqrt{m_{i}^{2}+\vec{k}_{i}^{2}}\right)^{-a} \frac{d \mu\left(m_{i}^{2}\right)}{\sqrt{m_{i}^{2}+\vec{k}_{i}^{2}}}\right)\left|g\left(\sum_{1}^{r} \vec{k}_{i}\right)\right|^{2} \prod_{1}^{r} d \vec{k}_{i} .
\end{aligned}
$$

Now

$$
\begin{aligned}
& \int\left(1+\sqrt{m_{i}^{2}+\vec{k}_{i}^{2}}\right)^{-a} \frac{d \mu\left(m_{i}^{2}\right)}{\sqrt{m_{i}^{2}+\vec{k}_{i}^{2}}} \\
\leqq & \left(1+\left|\vec{k}_{i}\right|\right)^{-a_{1}} \int\left(1+m_{i}\right)^{-a_{2}} m_{i}^{-1} d \mu\left(m_{i}^{2}\right) \leqq \text { constant }\left(1+\left|\vec{k}_{i}\right|\right)^{-a_{1}}
\end{aligned}
$$

by condition (ii), if $a=a_{1}+a_{2}$, and we choose $a$ large enough so that $a_{2}$ can be chosen equal to $q$.

Thus we only have to bound

$$
\int \prod_{1}^{r}\left(1+\left|\vec{k}_{i}\right|\right)^{-a_{1}}\left|g\left(\sum_{1}^{r} \vec{k}_{i}\right)\right|^{2} \prod_{1}^{r} d \vec{k}_{i}=\int \underbrace{\alpha * \cdots * \alpha}_{r}(\vec{p})|g(\vec{p})|^{2} d \vec{p},
$$

by Fubini's theorem, where $\alpha=(1+|\vec{p}|)^{-a}$.

If now we choose $a$ large enough so that $a_{1}$ is sufficiently large for $\alpha \in L_{p}\left(\boldsymbol{R}^{n}\right)$ for all $p \geqq 1$, by the Young inequality

$$
\alpha * \cdots * \alpha \in L_{\infty}\left(\boldsymbol{R}^{n}\right),
$$

and thus, as $g \in L_{2}\left(\boldsymbol{R}^{n}\right)$, we get a bound for the above integral.

In region II, $\left[k_{0}\right]_{s} \leqq 2\left[k_{0}\right]_{r-s}$, so the integral in question is bounded by $c^{\prime \prime \prime} \int_{\left[k_{0}\right]_{s} \leq 2\left[k_{0}\right]_{r-s}} \beta_{s}\left(k^{\prime}\right)^{d} \beta_{r-s}\left(k^{\prime \prime}\right)^{-2 b} d \rho_{+}(k)$, where $c^{\prime \prime \prime}$ is a suitable constant, as $g$ and $h$ are bounded. This is

$$
\begin{aligned}
& \leqq c^{\prime \prime \prime} \int_{\left[k_{0}\right]_{s} \leq\left[k_{0}\right]_{r-s}} \beta_{r-s}\left(k^{\prime \prime}\right)^{-2 b+d} d \rho_{+}(k) \\
& =c^{\prime \prime \prime} \int_{r-8}\left(k^{\prime \prime}\right)^{-2 b+d}\left(\prod_{1}^{s} \int_{\left|\overrightarrow{k_{i}}\right| \leqq 2\left[k_{0}\right]_{r-s}} \int_{0}^{2\left[k_{0}\right]_{r-s}} \frac{d \overrightarrow{k_{i}}}{\sqrt{m_{i}^{2}+\vec{k}_{i}^{2}}} d \mu\left(m_{i}^{2}\right)\right) d \rho^{+}\left(k^{\prime \prime}\right) \\
& \leqq c^{\prime \prime \prime} \int \beta_{r-s}\left(k^{\prime \prime}\right)^{-2 b+d}\left(\left(4\left[k_{0}\right]_{r-s}\right)^{n} \int_{0}^{2\left[k_{0}\right]_{r-s} s} \frac{d \mu\left(m^{2}\right)}{m}\right)^{s} d \rho_{+}\left(k^{\prime \prime}\right),
\end{aligned}
$$

which, if we use (iii), can be bounded by 


$$
c^{(i v)} \int \beta_{r-s}\left(k^{\prime \prime}\right)^{-2 b+d}\left[k_{0}\right]_{r-s}^{n+c_{2}} d \rho_{+}\left(k^{\prime \prime}\right) \leqq c^{(i v)} \int \beta_{r-s}\left(k^{\prime \prime}\right)^{-2 b+d+n+c_{2}} d \rho_{+}\left(k^{\prime \prime}\right),
$$

which we can bound, if we choose $b$ large enough, as in the proof of Lemma 2, independently of $m$ and $n$. End of proof.

Our next theorem looks at how these renormalized products transform under Lorentz transformations.

Proposition 3. Let $V$ be the natural representation of the Poincaré group $P$ (or inhomogeneous Lorentz group) by unitary or antiunitary operators in $\mathscr{H}$. Then, if $T \in P, \Gamma(V(T)) \mathscr{D}_{\infty}\left(H_{0}\right) \subset \mathscr{D}_{\infty}\left(H_{0}\right)$

Proof. $H_{0}=d \Gamma(B)$, where $B$ acts on $\mathscr{H}_{+}$as multiplication either by $k_{0}$ or $1+k_{0}$. Let $w \in \mathscr{D}_{\infty}(H) \cap \mathscr{K}_{m}, w \sim F\left(k_{1}, \cdots, k_{m}\right)$. Let $T \in P$. Then $T=\tau \Lambda$, where $\tau$ is a translation and $\Lambda$ a homogeneous Lorentz transformation. Writing $\Gamma(T)$ for $\Gamma(V(T))$, we have

$$
\left\|H_{0}^{a} \Gamma(T) w\right\|^{2}=\int \beta_{m}(k)^{2 a}\left|F\left( \pm \Lambda^{-1} k_{1}, \cdots, \pm \Lambda^{-1} k_{m}\right)\right|^{2} d \rho_{+}(k),
$$

$k=\left(k_{1}, \cdots, k_{n}\right)$, which is equal to

$$
\begin{aligned}
& \int \beta_{m}\left( \pm \Lambda k_{1}, \cdots, \pm \Lambda k_{m}\right)^{2 a}\left|F\left(k_{1}, \cdots, k_{m}\right)\right|^{2} d \rho_{+}(k) \\
\leqq & C \int \beta_{m}\left(k_{1}, \cdots, k_{m}\right)^{2 a s}\left|F\left(k_{1}, \cdots, k_{m}\right)\right|^{2} d \rho_{+}(k)=C\left\|H_{0}^{a s} w\right\|^{2}
\end{aligned}
$$

for some integer $s$, depending only on $\Lambda$, as $\beta( \pm \Lambda k)= \pm(\Lambda k)_{0}$ or $1 \pm(\Lambda k)_{0}$ on the support of $\rho_{+}$, and thus is a polynomial in $k$ and so can be bounded by a constant times a power of $\beta(k)$, say $\beta(k)^{s}$. End of proof.

THEROREM 3. Let $T$ be an inhomogeneous Lorentz transformation. Then, for every $\partial_{1}, \cdots, \partial_{r}$,

(a) for all $x \in M, \varphi\left(\partial_{1}, \cdots, \partial_{r} ; x\right)\left(\Gamma(T)^{-1} u, \Gamma(T)^{-1} w\right)=\varphi\left(\partial_{1}, \cdots, \partial_{r}\right.$; $T x)(u, w)$ for all $u, w \in \mathscr{D}_{\infty}\left(H_{0}\right)$.

(b) for all $f \in L_{1}(M), \quad \Phi\left(\partial_{1}, \cdots, \partial_{r} ; f\right)\left(\Gamma(T)^{-1} u, \Gamma(T)^{-1} w\right)=$ $\Phi\left(\partial_{1}, \cdots, \partial_{r} ; f_{T}\right)(u, w)$, for all $u, w \in \mathscr{D}_{\infty}\left(H_{0}\right)$, where $f_{T}(x)=f\left(T^{-1} x\right)$.

(c) If $\mathscr{D}_{\infty}\left(H_{0}\right) \subset \mathscr{D}\left(\Phi\left(\partial_{1}, \cdots, \partial_{r} ; f\right)\right)$, then $\mathscr{D}_{\infty}\left(H_{0}\right) \subset \mathscr{D}\left(\Phi\left(\partial_{1}, \cdots, \partial_{r}\right.\right.$; $\left.f_{T}\right)$ ), and $\Gamma(T) \Phi\left(\partial_{1}, \cdots, \partial_{r} ; f\right) \Gamma(T)^{-1} w=\Phi\left(\partial_{1}, \cdots, \partial_{r} ; f_{T}\right) w$ for all $w \in \mathscr{D}_{\infty}\left(H_{0}\right)$.

Proof. By Proposition 3, $\Gamma(T)^{-1} \mathscr{D}_{\infty}\left(H_{0}\right) \subset \mathscr{D}_{\infty}\left(H_{0}\right)$, and so the theorem makes sense.

To prove (a), it is enough to show

$$
\Gamma(T): \partial_{1} \Phi(g) \cdots \partial_{r} \Phi(g): \Gamma(T)^{-1}=: \partial_{1} \Phi\left(g_{T}\right) \cdots \partial_{r} \Phi\left(g_{T}\right):
$$


which follows from $\Gamma(T) \Phi(g) \Gamma\left(T^{-1}\right)=\Phi\left(g_{T}\right)$, and the fact that the renormalization operator : : is invariant under transformations by an operator of the form $\Gamma(V)$, where $V$ is a unitary operator in $\mathscr{H}$, since $\Gamma(V)$ preserves commutation relations and vacuum expectations, in terms of which : : is uniquely defined (see [4]).

Since if $g \rightarrow \delta(\cdot-x), g_{T} \rightarrow \delta(\cdot-T x)$, (a) follows from the explicit construction in the proof of Theorem 1. (b) follows from (a), and (c) follows from (b) plus the fact that $\left\|\Gamma(T)^{-1} u\right\|=\|u\|$. End of proof.

COROLLARY. Let $f$ be a real-valued function in $L_{1}(M)$ such that $\mathscr{D}_{\infty}\left(H_{0}\right) \subset \mathscr{D}\left(\Phi\left(\partial_{1}, \cdots, \partial_{r} ; f\right)\right)$. Then, if there exists a translation $\tau$ and homogeneous Lorentz transformation $\Lambda$ which preserves the direction of time, such that $f_{\tau A}$ is an even function on the time variable, $\Phi\left(\partial_{1}, \cdots, \partial_{r} ; f\right) \mid \mathscr{D}_{\infty}\left(H_{0}\right)$ admits a self-adjoint extension.

Proof. It is clear from the explicit construction of $\Phi\left(\partial_{1}, \cdots, \partial_{r}\right.$; $f$ ) that it is a symmetric operator if $f$ is real. By last theorem (c), $\Phi\left(\partial_{1}, \cdots, \partial_{r} ; f\right)=\Gamma(\tau \Lambda)^{-1} \Phi\left(\partial_{1}, \cdots, \partial_{r} ; f_{\tau \Lambda}\right) \Gamma(\tau \Lambda)$, where $\Gamma(\tau \Lambda)$ is unitary, as $\Lambda$ preserves the direction of time. Thus it is enough to show $\Phi\left(\partial_{1}, \cdots, \partial_{r} ; f\right) \mid \mathscr{D}_{\infty}(H)$ admits a self-adjoint extension, if $f$ is even in time. Let $\mathscr{X}$ be time reversal, i.e. $\mathscr{X}(x, t)=(x,-t)$. Then $\Gamma(\mathscr{X})$ is an antiunitary operator on $\mathscr{K}$, such that

$$
\Gamma(\mathscr{X})^{2}=\Gamma\left(\mathscr{X}^{2}\right)=\Gamma(I)=I,
$$

so $\Gamma(\mathscr{X})$ is a conjugation on $\mathscr{K}$. By Theorem 3 (c),

$$
\begin{aligned}
\Gamma(\mathscr{X}) \Phi\left(\partial_{1}, \cdots, \partial_{r} ; f\right) \Gamma(\mathscr{H})^{-1} w & =\Phi\left(\partial_{1}, \cdots, \partial_{r} ; f_{\mathscr{Z}}\right) w \\
& =\Phi\left(\partial_{1}, \cdots, \partial_{r} ; f\right) w
\end{aligned}
$$

for all $w \in \mathscr{D}_{\infty}\left(H_{0}\right)$, as $f$ is even in time, and so,

$$
\Phi\left(\partial_{1}, \cdots, \partial_{r} ; f\right) \mid \mathscr{D}_{\infty}\left(H_{0}\right),
$$

being a densely defined symmetric operator commuting with a conjugation, admits a self-adjoint extension. End of proof.

\section{REFERENCES}

1. J. M. Cook, The mathematics of second quantization, Trans. Amer. Math. Soc., 74 (1953), 222-245.

2. O. W. Greenberg, Generalized free fields and models of local field theory, Annals of Physics, 16 (1961), 158-176.

3. L. Schwartz. Theorie des Distributions, Vol. II, Hermann, Paris, 1959.

4. I. Segal, Nonlinear functions of weak processes I, J. Functional Analysis, 4 (1969), 404-456.

5. - Nonlinear functions of weak processes II, J. Functional Analysis, 6(1970), 29-75. 
6. I. Segal, Local Noncommutative Analysis, Problems in Analysis, edited by R. C. Gunning, Princeton University Press, (1970), 111-129.

7. A. S. Wightman, Some results on the structure of relativistic quantum field theory, Proc. Int'l. Cong. of Math., Stockholm, (1962), 587-595.

8. A. S. Wightman and L. Gårding, Fields as operator-valued distributions in relativistic quantum field theory, Arkiv för Fysik, 28 (1964), 129-184.

Received November 12, 1971. The contents of this paper were part of the author's $\mathrm{Ph}$. D. thesis at M.I.T. The author wishes to express his gratitude to Professor Irving Segal, for having introduced him to this research, and taken a continuing interest in its progress. The author is also in debt to Niels Skovhus Poulsen and Wilbert Wils for many helpful discussions.

UNIVERSity of CALIFornia, Los ANGELeS 


\section{PACIFIC JOURNAL OF MATHEMATICS}

EDITORS

\author{
H. SAMELSON \\ Stanford University \\ Stanford, California 94305 \\ C. R. HOBBY \\ University of Washington \\ Seattle, Washington 98105
}

\author{
J. DUGUNDJI \\ Department of Mathematics \\ University of Southern California \\ Los Angeles, California 90007 \\ RICHARD ARENS \\ University of California \\ Los Angeles, California 90024
}

\section{ASSOCIATE EDITORS}

E. F. BECKENBACH

B. H. NeUManN

F. WOLF

K. YosHIDA

\section{SUPPORTING INSTITUTIONS}

UNIVERSITY OF BRITISH COLUMBIA

CALIFORNIA INSTITUTE OF TECHNOLOGY

UNIVERSITY OF CALIFORNIA

MONTANA STATE UNIVERSITY

UNIVERSITY OF NEVADA

NEW MEXICO STATE UNIVERSITY

OREGON STATE UNIVERSITY

UNIVERSITY OF OREGON

OSAKA UNIVERSITY
UNIVERSITY OF SOUTHERN CALIFORNIA

STANFORD UNIVERSITY

UNIVERSITY OF TOKYO

UNIVERSITY OF UTAH

WASHINGTON STATE UNIVERSITY UNIVERSITY OF WASHINGTON

AMERICAN MATHEMATICAL SOCIETY NAVAL WEAPONS CENTER 


\section{Pacific Journal of Mathematics}

\section{Vol. 45, No. $1 \quad$ September, 1973}

William George Bade, Complementation problems for the Baire classes .......... 1

Ian Douglas Brown, Representation of finitely generated nilpotent groups ........ 13

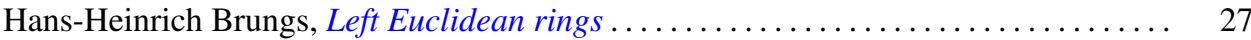

Victor P. Camillo and John Cozzens, A theorem on Noetherian hereditary rings ..... 35

James Cecil Cantrell, Codimension one embeddings of manifolds with locally flat

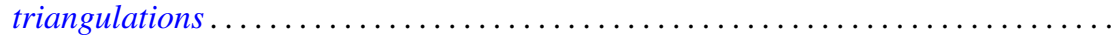

L. Carlitz, Enumeration of up-down permutations by number of rises . . . . . . . . . .

Thomas Ashland Chapman, Surgery and handle straightening in Hilbert cube

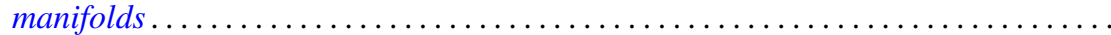

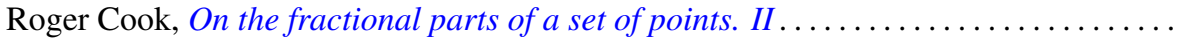

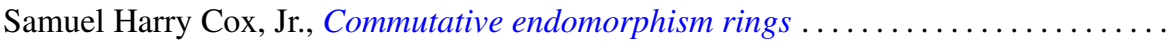

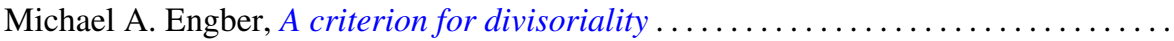

Carl Clifton Faith, When are proper cyclics injective . . . . . . . . . . . . . . 97

David Finkel, Local control and factorization of the focal subgroup . . . . . . . . . 113

Theodore William Gamelin and John Brady Garnett, Bounded approximation by

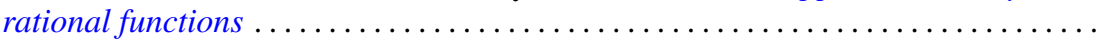

Kazimierz Goebel, On the minimal displacement of points under Lipschitzian

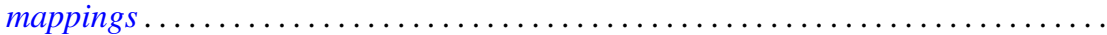

Frederick Paul Greenleaf and Martin Allen Moskowitz, Cyclic vectors for representations associated with positive definite measures: nonseparable

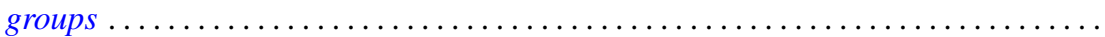

Thomas Guy Hallam and Nelson Onuchic, Asymptotic relations between perturbed linear systems of ordinary differential equations .

David Kent Harrison and Hoyt D. Warner, Infinite primes of fields and completions. .

James Michael Hornell, Divisorial complete intersections . ......

Jan W. Jaworowski, Equivariant extensions of maps ..............

John Jobe, Dendrites, dimension, and the inverse arc function .. .

Gerald William Johnson and David Lee Skoug, Feynman integrals of non-factorable

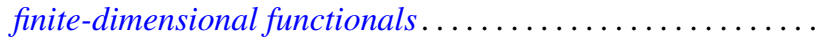

Dong S. Kim, A boundary for the algebras of bounded holomorphic functions ...... 269

Abel Klein, Renormalized products of the generalized free field and its derivatives ... 275

Joseph Michael Lambert, Simultaneous approximation and interpolation in $L_{1}$ and

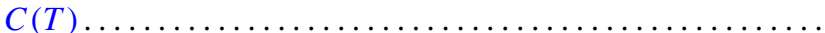

Kelly Denis McKennon, Multipliers of type $(p, p)$ and multipliers of the group

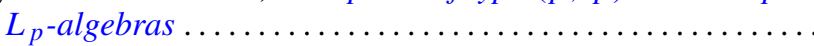

William Charles Nemitz and Thomas Paul Whaley, Varieties of implicative

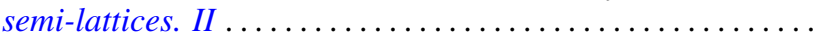

Donald Steven Passman, Some isolated subsets of infinite solvable

Norma Mary Piacun and Li Pi Su, Wallman compactifications on E-completely

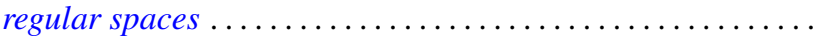

Jack Ray Porter and Charles I. Votaw, $S(\alpha)$ spaces and regular Hausdorff extensions....

Gary Sampson, Two-sided $L_{p}$ estimates of convolution transforms .

Ralph Edwin Showalter, Equations with operators forming a rig
Raymond Earl Smithson, Fixed points in partially ordered sets .

Victor Snaith and John James Ucci, Three remarks on symmetric products and

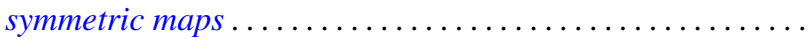

